Revista de Derecho

\title{
Análisis de clústers jurisprudenciales en el área andina: acercamiento mixto al ius constitutionale commune
}

Analysis of Jurisprudential Clusters in the Andean Area:
Mixed Approach to the lus Constitutionale Commune

Efrén Ernesto Guerrero Salgado

Profesor de la Pontificia Universidad Católica del Ecuador

Quito, Ecuador

eeguerrero@puce.edu.ec

ORCID: 0000-0003-4636-0362

Artículo de investigación

DOI: https://doi.org/10.32719/26312484.2021.36.9

Fecha de recepción: 4 de enero de 2021

Fecha de revisión: 19 de enero de 2021

Fecha de aceptación: 16 de marzo de 2021

Fecha de publicación: 1 de julio de 2021 


\section{RESUMEN}

El presente documento propone un acercamiento de tipo mixto a la jurisprudencia de tres cortes constitucionales americanas, respecto al uso de jurisprudencia vinculante emitida desde el Sistema Interamericano de Derechos Humanos (SIDH), con el de demostrar empíricamente la existencia de un lus Constitucionale Commune. Este concepto es un fenómeno de construcción de una comunidad jurídica alrededor de la Convención Americana de Derechos Humanos y el corpus iuris interamericano, sostenida en la idea de estándares provenientes del derecho internacional y aprovechadas por las Altas Cortes. Este texto explora esa posibilidad, mediante un levantamiento de información cuali-cuantitativa de la jurisprudencia de las Cortes Constitucionales peruana, colombiana y ecuatoriana durante el año 2020, procesadas por la metodología del análisis de redes sociales (social network análisis). Los datos obtenidos demuestran: a) un uso más o menos constante la jurisprudencia del SIDH; y b) la posibilidad fáctica de este fenómeno como un elemento que puede ser estudiado en nuestra región como una muestra de una auténtica estructura integracionista del derecho constitucional.

Palabras Clave: jurisprudencia, política pública, derecho constitucional, análisis de redes sociales, derecho comparado, derecho constitucional, ius constitutionale commune, métodos mixtos.

\section{ABSTRACT}

This document proposes a mixed type approach to the jurisprudence of three American constitutional courts, regarding the use of binding jurisprudence issued from the Inter-American Human Rights System (SIDH), in order to empirically demonstrate the existence of a Ius Constitucionale Commune. This concept is a phenomenon of construction of a legal community around the American Convention on Human Rights and the inter-American corpus iuris, supported by the idea of standards derived from international law and taken advantage of by the High Courts. This text explores this possibility, through a survey of qualitativequantitative information on the jurisprudence of the Peruvian, Colombian and Ecuadorian Constitutional Courts during 2020, processed by the methodology of social network analysis (social network analysis). The data obtained demonstrate: a) a more or less constant use of the jurisprudence of the IAHRS; and b) the factual possibility of this phenomenon is an element that can be studied in our region as a sample of an authentic integrationist structure of constitutional law.

KEYWORDS: jurisprudence, public policy, constitutional law, analysis of social networks, comparative law, constitutional law, ius constitutionale commune, mixed methods. 


\section{INTRODUCCIÓN: GLOBALIZACIÓN DEL DERECHO Y CONFLUENCIAS DE APLICACIÓN DE UN DERECHO COMÚN}

E 1 Derecho Internacional se encuentra en una encrucijada: en un mundo de riesgo global, se debe construir un sistema de orden, que a pesar de la anarquía ${ }^{1}$ debe dar respuestas a interrogantes similares para todos los Estados. ${ }^{2}$ La primera es la legislación: ¿Cuáles son las obligaciones, poderes y procedimientos relevantes del derecho internacional público?, la segunda se refiere al cumplimiento: ¿Las obligaciones internacionales pueden cumplirse?, y la última se refiere a la institucionalidad: ¿Qué estructuras organizacionales logran que esas reglas se cumplan?

La mejor respuesta posible a esas tres preguntas descansa en el corpus iure internacional. Los Estados no pueden actuar de manera rebelde dentro del sistema, ${ }^{3}$ sino ser interdependientes, para buscar cierta gobernanza global. En ese sentido, los sujetos de derecho internacional se relacionan a través del sistema de fuentes previsto en el art. 38 del estatuto de la CIJ que da origen, a su vez, a derechos recíprocos para ella o ellas. ${ }^{4}$

En esa búsqueda de reglas equivalentes, el sistema de derechos humanos ha sido el medio de equiparar valores con mínimos de acción compartidos por la comunidad internacional. Este hecho es todavía limitado, pues no se puede perforar por completo la soberanía del Estado para anular las costumbres y normas que reflejan los valores morales locales. ${ }^{5}$ Entonces, la solución planteada es aprovechar las relaciones entre el derecho internacional y el derecho interno. En los sistemas regionales, con mayor éxito en las construcciones de prácticas equivalentes en materia de protección ${ }^{6}$ se ha actuado de manera táctica en ese tema, debido a la tensión entre el objetivo de expandir

1. Ver Henry Kissinger, Orden Mundial (Barcelona: Debate, 2016).

2. Ver Armin von Bogdandy y Pedro Villarreal, International Law on Pandemic Response: A First Stocktaking in Light of the Coronavirus Crisis (Heidelberg: Max Planck Institute for Comparative Public Law \& International Law, MPIL, 2020).

3. Robert Keohane y Joseph Nye, Power and Interdependence. World Politics in Transition (Boston: Little Brown and Company, 1977).

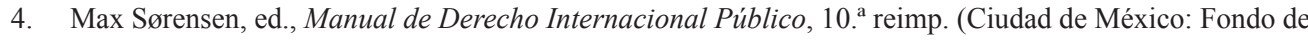
Cultura Económica, 2008), 201. Esto incluye a actores no estatales cuya participación se vuelve crítica para el control del flujo global de estructuras normativas. Ver Augusto Lopez-Claros, Arthur Dahl y Maja Groff, Global Governance and the Emergence of Global Institutions for the 21st Century (Nueva York: Cambridge University Press, 2020).

5. Jootaek Lee, "Paradox of Hierarchy and Conflicts of Values: International Law, Human Rights, and Global Governance", Northwestern University Journal of International Human Rights, vol. 18 (1) (2019): 92.

6. Ibíd., 92 . 
derechos y la necesidad de ganar legitimidad con las circunscripciones nacionales. ${ }^{7}$ La evidencia indica que:

Las obligaciones derivadas de dicha normativa internacional deben ser tomadas en cuenta, según lo dispuesto en el artículo 29.b) de la Convención, pues quienes se hallan protegidos por el régimen de dicho instrumento no pierden por ello los derechos o facultades que ya tengan conforme a la legislación del Estado bajo cuya jurisdicción se encuentran, sino se complementan o integran para precisar su alcance o determinar su contenido. ${ }^{8}$

En el caso del Sistema Interamericano de Protección de Derechos Humanos (SIDH), la Comisión y la Corte han tenido efectivamente en cuenta ese hecho: mediante de la jurisprudencia, y tomando en cuenta su entorno político, mantienen su legitimidad y la probabilidad de amplia aceptación de sus decisiones. De hecho, uno de los mecanismos en los que la Corte tiene mayor éxito, es que su jurisprudencia sea aceptada y usada por sus contrapartes las Cores Constitucionales Nacionales. Con esos antecedentes, un sector de la doctrina propone una "columna vertebral" de elementos comunes en los ordenamientos latinoamericanos, producto de la interacción entre la Altas Cortes Nacionales y el SIDH. Según estos autores, es "el enfoque más ambicioso y con mayor proyección de los que actualmente propugnan en la región un constitucionalismo transformador en el contexto de la Inter americanización del derecho". ${ }^{9}$ En este sentido, las Cortes más importantes de la región, al utilizar la jurisprudencia del SIDH, generarían una "cultura común o ius commune que en materia de democracia induciría al reconocimiento de ciertos principios y valores comunes entre los Estados del hemisferio". ${ }^{10}$

Considerando que deberían existir, mediante el uso del bloque de constitucionalidad, elementos concurrentes en las diferentes tradiciones constitucionales latinoamericanas, el motivo del presente texto es hacer un análisis mediante métodos mixtos de

7. Ver Wayne Sandholtz y Mariana Rangel Padilla, "Law and Politics in the Inter-American System: The Amnesty Cases", Journal of Law and Courts 8 (1) (2020): 10.

8. Corte IDH, "Sentencia de 15 de septiembre de 2005 (Fondo, Reparaciones y Costas)", Caso de la "Masacre de Mapiripán" vs. Colombia, 15 de septiembre de 2005, párr. 115.

9. Leonardo García Jaramillo, "De la 'constitucionalización' a la "convencionalización” del ordenamiento jurídico. La contribución del ius constitutionale commune”, Revista Derecho del Estado, n. ${ }^{\circ} 36$ (2016): 132, doi:10.18601/01229893.n36.05.

10. Mariela Morales Antoniazzi, “¿La democracia como principio del ius constitutionale commune en América Latina? Construcción, reconstrucción y desafíos actuales para la justicia constitucional”, en Ius Constitutionale Commune en América Latina. Texto básico para su comprensión, coord. Armin von Bogdandy, Mariela Morales Antoniazzi y Eduardo Ferrer Mac-Gregor (Querétaro: Max Planck Institute for Comparative Public Law and International Law, 2017), 417-57. 
investigación, ${ }^{11}$ para detectar la existencia fáctica de esa jurisprudencia compartida; y analizar brevemente el probable valor jurídico de esta. Para esto, se hará un análisis mixto de la jurisprudencia de tres países latinoamericanos que aceptan la presencia del bloque de constitucionalidad y el valor de la jurisprudencia en su ordenamiento, con la metodología de las redes sociales, ${ }^{12}$ que cuenta con el poder explicativo suficiente para visualizar gráfica y matemáticamente la red de conexiones entre las diferentes piezas jurisprudenciales.

En este sentido, el presente texto se organiza de la siguiente forma: la primera sección explicará los alcances del ius constitutionale commune en el sentido establecido por el SIDH, y los alcances del concepto en el marco de la jurisprudencia de la Cortes Constitucionales de Colombia, Ecuador y Perú. En segundo lugar, se explicarán los límites del concepto. Tercero, se explicará el modelo de análisis que se propondrá para la jurisprudencia, centrado en una proyección de las relaciones el derecho de los jueces del SIDH con la nacional. Posteriormente, se hará una discusión de datos obtenidos, en relación con la literatura existente, para finalmente realizar conclusiones y recomendaciones a partir de la data.

\section{EL IUS CONSTITUTIONALE COMMUNE AMERICANO: ALCANCES Y POSIBILIDADES}

Los Estados como sujetos de derecho internacional en el marco de decisiones de racionalidad limitada, ${ }^{13}$ actúan con heurísticas que ayudan a aumentar su comprensión de los fenómenos y que disminuyen la incertidumbre. ${ }^{14}$ Con esos antecedentes, teóricos del derecho internacional proponen la creación de un ius commune: una aproximación conceptual para desarrollar un derecho colaborativo, articulado y armonizado a nivel supranacional y local". ${ }^{15} \mathrm{La}$ doctrina propone que existen una serie de fenómenos en el manejo del derecho internacional que aseguran esa comunicación:

a) Normas "de apertura" en los diferentes ordenamientos jurídicos, en los que los tratados internacionales se encuentran: a) en un nivel superior dentro del ordena-

11. Burke Johnson, Anthony Onwuegbuzie y Lisa Turner, "Toward a Definition of Mixed Methods Research", Journal of Mixed Methods Research, vol. 1 (2) (2007): 112-33, doi:10.1177/1558689806298224.

12. Stanley Wasserman y Katherine Faust, Social network analysis: Methods and application (Nueva York: Cambridge University Press, 1994).

13. Ver Russell Korobkin y Thomas Ulen, "Law and behavioral science: Removing the rationality assumption from law and economics", California Law Review, vol. 88 (2000): 1053-66, doi:10.2139/ssrn.229937.

14. Georg Jellinek, Teoría General del Estado (Buenos Aires: Albatros, 1978), 133.

15. Juan Pablo Pampillo Baliño, "Derecho comunitario y ius commune americano: dos asignaturas pendientes para las ciencias jurídicas de nuestro continente”, Foro: Revista de Derecho, n. ${ }^{\circ} 18$ (2017): 8. 
miento jurídico; o b) generan bloque de constitucionalidad, ${ }^{16}$ por lo que los tratados internacionales en materia de derechos humanos se integran dentro de una red de herramientas de aplicación directa, de forma expresa o como un principio.

b) Existencia de un patrón más general de creciente "comunicación judicial transnacional" o "globalización judicial". ${ }^{17}$

c) Entonces al tener: a) cláusulas de apertura en su ordenamiento jurídico; y b) movimiento de ideas entre jurisdicciones, con elementos sustantivos y de valores comunes manifestados en sentencias. Respecto a este punto la Corte Interamericana ha afirmado, que:

a) Los tratados de derechos humanos son instrumentos vivos, cuya interpretación tiene que acompañar la evolución de los tiempos y las condiciones de vida actuales.

b) Ese tipo de interpretación, que se llama evolutiva es consecuente con las reglas generales de interpretación establecidas en el artículo 29 de la CADH. ${ }^{18}$

c) La Convención de Viena sobre el Derecho de los Tratados, especialmente el párrafo tercero del artículo 31, autoriza la utilización de medios interpretativos adecuados.

Entonces, en el escenario de que sucedan los hechos anteriormente descritos, se propone el siguiente esquema que busca explicar el funcionamiento del fenómeno:

16. El bloque de constitucionalidad se refiere a aquellas normas y principios que, sin aparecer formalmente en el articulado del texto constitucional, son utilizados como parámetros del control de constitucionalidad de las leyes, por cuanto han sido normativamente integrados a la Constitución, por diversas vías y por mandato de la propia Constitución. Véase en el caso ecuatoriano la Sentencia n. ${ }^{\circ} 132-16-S E P-C C$, del 20 de abril de 2016, MP: DR. Principales WMA Wendy Molina Andrade, Registro Oficial n. ${ }^{\circ} 799$, Suplemento, 18 de julio de 2016 de la Corte Constitucional, y en su homóloga colombiana, en las sentencias C-225-95 MP: Alejandro Martínez Caballero.

17. Ver Paolo Carozza, "'My Friend is a Stranger': The Death Penalty and the Global Ius Commune of Human Rights", Texas Law Review, vol. 81 (2003): 1031-86, url: https://scholarship.law.nd.edu/law_faculty_scholarship/536.

18. Artículo 29. Normas de Interpretación.- Ninguna disposición de la presente Convención puede ser interpretada en el sentido de:

a) permitir a alguno de los Estados partes, grupo o persona, suprimir el goce y ejercicio de los derechos y libertades reconocidos en la Convención o limitarlos en mayor medida que la prevista en ella;

b) limitar el goce y ejercicio de cualquier derecho o libertad que pueda estar reconocido de acuerdo con las leyes de cualquiera de los Estados partes o de acuerdo con otra convención en que sea parte uno de dichos Estados;

c) excluir otros derechos y garantías que son inherentes al ser humano o que se derivan de la forma democrática representativa de gobierno, y

d) excluir o limitar el efecto que puedan producir la Declaración Americana de Derechos y Deberes del Hombre y otros actos internacionales de la misma naturaleza. 


\section{Ilustración 1. Proceso teórico del Ius Commune}

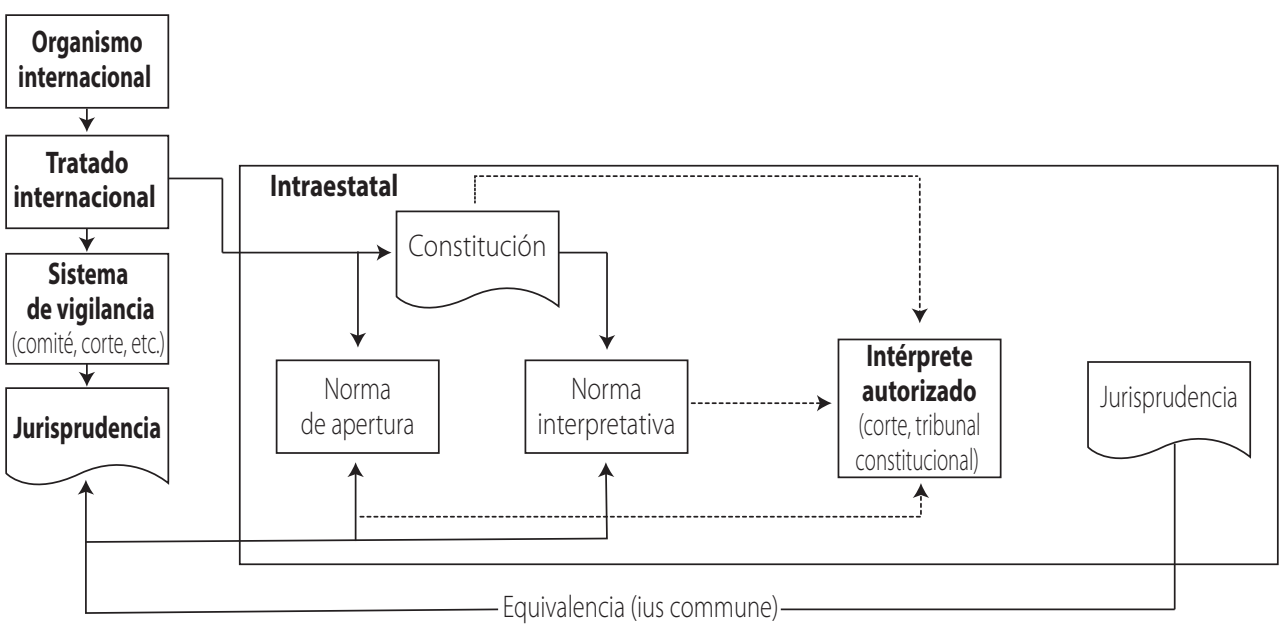

Fuente y elaboración propias.

En ese sentido la jurisprudencia, al ser una interpretación autorizada del tratado, debería ser vinculante para los Estados parte; en consecuencia, cuando un Estado es parte de un tratado internacional, todos sus órganos, incluidos sus jueces, están sometidos a aquél. Dentro de cada Estado debería existir una disposición constitucional que regule el uso directo de los tratados internacionales y de su corpus iure. ${ }^{19}$ Finalmente, debería existir un intérprete autorizado que sea capaz de generar esa "conexión" entre ambos ordenamientos y establecer esos límites. ${ }^{20} \mathrm{Al}$ respecto, la Corte Constitucional ecuatoriana señala que:

La tarea de interpretación constitucional, por mandato constitucional, si bien puede ser efectuada por todos quienes la leen y la aplican - incluidos los órganos de administración de justicia constitucional-, cuando constituye interpretación auténtica del texto constitucional, está reservada para el Pleno de la Corte Constitucional, como máximo tribunal competente. ${ }^{21}$

19. Corte IDH, "Sentencia de 5 de febrero de 2018 (Excepciones Preliminares, Fondo, Reparaciones y Costas)", Caso Pueblo Indigena Xucuru y sus miembros vs. Brasil, 5 de febrero de 2018, https://www.cortei dh.or.cr/docs/casos/articulos/seriec_346_esp.pdf.

20. García Jaramillo, "De la 'constitucionalización' a la 'convencionalización' del ordenamiento jurídico...".

21. Ecuador Corte Constitucional del Ecuador, "Sentencia n. ${ }^{\circ}$ 132-16-SEP-CC", Caso n. ${ }^{\circ}$ 1264-15-EP, 20 de abril de 2020. 
Este fenómeno (de suceder) tiene consecuencias importantes a nivel sociopolítico e indican que "existe un verdadero - aunque no seguro- espacio judicial - $\mathrm{y}$ convencional - latinoamericano en materia de derechos humanos", asumiendo que los Estados parte del SIDH comulgan con la existencia de esta idea. ${ }^{22}$ Se proponen consecuencias importantes: no solo hablar de estándares equivalentes, sino "un constitucionalismo regional de los derechos con garantías supranacionales". ${ }^{23}$ Los autores lo citan en Latinoamérica y en el SIDH, gracias al avance de las sentencias de la Corte IDH como referente de protección de garantías constitucionales en la región:

La comprensión legal de la Corte Interamericana de Derechos Humanos cambia cuando se parte de la idea de una institución internacional (...) a la idea de que es la institución que más nutre y alimenta la red latinoamericana del constitucionalismo transformativo. Dicha red conecta la Corte IDH a las cortes nacionales y otros tribunales internos. ${ }^{24}$

En suma, la doctrina supone el fenómeno de Ius Commune latinoamericano con la existencia de cláusulas combinadas de apertura para la construcción de valores en común entre Altas Cortes nacionales, como centro epistémico la jurisprudencia del SIDH. Esto tiene un particular interés, debido a la creciente constitucionalización, relaciones jurídicas, y el creciente papel de los jueces constitucional como operadores erga omnes. ${ }^{25}$ Esto obliga a repensar la cultura jurídica que se construye en torno a la aplicación normativa en el marco del derecho positivo. ${ }^{26}$ De las críticas al presente concepto, se referirá la siguiente sección.

22. Sergio García Ramírez, "La 'navegación americana’ de los derechos humanos: hacia un Ius Commune", en Ius Constitutionale Commune en América Latina. Texto básico para su comprensión, coord. Armin von Bogdandy, Mariela Morales Antoniazzi y Eduardo Ferrer Mac-Gregor (Querétaro: Max Planck Institute for Comparative Public Law and International Law, 2017), 55-190.

23. Armin von Bogdandy, "Ius Constitutionale Commune in Latin America: A Look at a Transformative Constitutionalism", Revista Derecho del Estado, n. ${ }^{\circ} 34$ (2015): 3-50.

24. Ana Santano, "Derechos humanos para el desarrollo de una sociedad realmente globalizada", Opinión Jurídica 19 (38) (2020): 39-57.

25. Ney Molero Martínez, "Justicia constitucional y democracia: casos Colombia, Ecuador y Venezuela", Revista Digital de Investigación y Postgrado 2 (3) (2021): 89-100.

26. Manuel Aragón Reyes, "Dos problemas falsos y uno verdadero: 'neoconstitucionalismo', 'garantismo' y aplicación judicial de la Constitución”, Cuestiones Constitucionales, n. ${ }^{\circ} 29$ (2015): 3-25, doi:10.1016/ s1405-9193(13)71288-5. 


\section{CRÍTICAS AL CONCEPTO: LA SOBERANÍA COMO LÍMITE DEL DERECHO INTERNACIONAL}

Este concepto tiene una serie de límites, entendidos en el marco de la aplicación de la voluntad soberana del Estado para comprometerse internacionalmente: si se puede ejercer el poder constituyente para cambiar la base de su ordenamiento interno, al organizarse de forma supra estatal, pasa a tener presente otro ordenamiento (el internacional) que no depende de su voluntad soberana. ${ }^{27}$ Esto supone la construcción de un sistema armónico mediante y de acuerdo con los tratados internacionales como medio de evitar inconstitucionalidad. ${ }^{28}$

Por otro lado, hay resistencia en instancias jurisdiccionales a aplicar el SIDH, basados en la idea del "margen de apreciación", que permite una adaptación "con una importante maniobrabilidad del Estado en su adaptación". ${ }^{29}$ Eso sí, el SIDH, al contrario del sistema europeo, ha limitado el ejercicio de ese derecho y es una figura residual limitada a escenarios, en los que las particularidades propias de cada país no afecten el contenido de derecho ${ }^{30} \mathrm{o}$ el bien jurídico protegido. ${ }^{31}$ Pese a estas críticas, los países latinoamericanos en su mayoría indican cláusulas de apertura de entrada del derecho internacional, con valor vinculante y superior, formado por un conjunto de instrumentos de contenido y efectos jurídicos variados, ${ }^{32}$ fuera de la Constitución formal pero que comparten con ella su máxima jerarquía y sirven como parámetros de normas inferiores. ${ }^{33}$

Por otro lado, hay otros autores que suponen ciertas fallas de origen. Por ejemplo, Conde y Luque propone que "no se puede legislar en la sociedad internacional de

27. Precisamente esto es consecuencia de la intervención de otros sujetos iguales en derechos y obligaciones, tal como lo establece el artículo 2 de la Carta de las Naciones Unidas al hablar del "principio de la igualdad soberana".

28. Rafael Oyarte, Derecho Constitucional (Quito: Corporación de Estudios y Publicaciones, 2015).

29. Francisco Barbosa Delgado, "Los límites a la doctrina del margen nacional de apreciación en el Tribunal Europeo y la Corte Interamericana de derechos humanos: intervención judicial en torno a ciertos derechos de las minorías étnicas y culturales", Revista Derecho del Estado, n. ${ }^{\circ} 26$ (2011): 107-35, url: https://revistas.uexternado.edu.co/index.php/derest/article/view/2881.

30. Pablo Sánchez-Molina, "Margen de apreciación nacional (en los sistemas de protección internacional de los derechos humanos)", EUNOMÍA. Revista en Cultura de la Legalidad, n. 9 (2016): 224-31.

31. Esto es patente en el caso Castañeda Guzmán, en el cual la Corte IDH otorga un margen de apreciación a la hora de imponer requisitos para ejercitar los derechos políticos.

32. Corte IDH, "Sentencia de 20 de octubre de 2016 (Excepciones Preliminares, Fondo, Reparaciones y Costas)", Caso Trabajadores de la Hacienda Brasil Verde vs. Brasil, 20 de octubre de 2016, párr. 247.

33. Lautaro Pittier y Ricardo Rincón, "La evolución de los DDHH en la Constitución. Diálogos y tensiones entre la Constitución Formal y la Constitución Material”, Revista Argentina de Derecho Público, n. 5 (2020). 
los Estados", ya que hay que entender los tratados como productos de otro espacio legislativo. ${ }^{34}$ Otros autores indican que el uso del derecho internacional es una "apreciación subjetiva, oportunista y discriminatoria de quienes en cada caso se encuentran en una posición de superioridad". ${ }^{35}$ Con este contexto, Castilla Juárez propone las siguientes limitaciones al Ius Constitucional Communne: ${ }^{36}$

a) No todos los países que se pueden ubicar como latinoamericanos son parte de la $\mathrm{CADH}$, solo lo son 18 de 20 .

b) De esos 18 al menos cuatro (Argentina, México, El Salvador y Uruguay) mantienen reservas y declaraciones interpretativas.

c) Solo 2 Estados (Costa Rica y Uruguay) son parte de todos los protocolos adicionales (2) a la CADH y tratados especializados.

d) Respecto a reservas y declaraciones interpretativas que tienen los siete tratados antes indicados, lo común llegaría a 9 de 20 países (Argentina, Costa Rica, Brasil, Ecuador, México, Nicaragua, Panamá, Paraguay y Uruguay).

En la hipótesis del autor, los únicos países latinoamericanos que son parte de todos los tratados interamericanos de derechos humanos, sin reservas ni declaraciones interpretativas y, por tanto, que sí tienen en principio un sistema normativo verdaderamente común, son Costa Rica, Ecuador, Panamá y Paraguay; y son los únicos que comparten — basándonos en esa teoría - un Ius Constitucionale Commune. En resumen, los críticos proponen hacer una reflexión desde las formas y otra desde el fondo de la aplicación normativa. De no existir límites al uso del derecho internacional, se pondría a los jueces en una elección racional "de intereses" y no de límites", respecto a los grandes temas nacionales, pues podrían hacer cualquier interpretación, basada en las ideas existentes. ${ }^{37}$ Además, si no se toma en cuenta la unidad y validez del sistema jurídico y la obligación de una interpretación holística del sistema, se vería todo el sistema constreñido a visiones subjetivas de la realidad. ${ }^{38}$

En opinión de quien suscribe, las críticas son parcialmente correctas. Aunque es cierto que el poder constituyente limita la aplicación de normas externas, ${ }^{39}$ existen elementos que muestran la necesidad de este derecho común. En primer lugar, una de-

34. Rafael Conde y Luque, Derecho internacional público (Sevilla: Athenaica Ediciones Universitarias, 2015), 61.

35. Antonio Remiro Brotóns, Derecho Internacional (Valencia: Tirant lo Blanch, 2007), 49.

36. Karlos Castilla Juárez, “¿Qué tan común es lo común del Ius Constitutionale Commune Latinoamericano?", Ventana Jurídica, 21 de junio de 2017, https://bit.ly/2KdFP2K.

37. Ramiro Ávila, El neoconstitucionalismo transformador. El Estado y el derecho en la Constitución de 2008 (Quito: Universidad Andina Simón Bolívar, Sede Ecuador, 2011), 54.

38. Ver Luigi Ferrajoli, Poderes salvajes: la crisis de la democracia constitucional (Madrid: Trotta, 2013).

39. Oyarte, Derecho Constitucional, 80. 
cisión soberana precisa decisión de los Estados en la forma de una personalidad funcional, capaz de usar sus competencias. ${ }^{40}$ En segundo lugar, sucede un fenómeno de "cesión de competencias", en el que la autoridad decide qué parte de su ámbito decisorio - en este caso la construcción epistémica en cierta materia decisoria- queda en manos de una organización internacional, con la consecuencia particular de que "las decisiones que adopten podrán tener, en determinadas ocasiones, autoridad directa e inmediata en los órdenes jurídicos nacionales, con carácter prevalente". ${ }^{41}$ Finalmente, las cortes constitucionales influyen en las decisiones futuras de diferentes maneras en diferentes tradiciones jurídicas y pueden verse influenciadas por las recientes tendencias jurisprudenciales y las modas en la jurisprudencia. ${ }^{42} \mathrm{El}$ momento que se da esa cesión del poder soberano del Estado, implica que existe un gradual ejercicio común de aceptación, y que depende de la técnica de cada país, basado en el bloque de constitucionalidad como una estructura suplementaria de medición de la constitucionalidad de los actos del Estado, y el control de convencionalidad como mecanismo reforzado de cumplimiento obligatorio. Debe tenerse presente que para autores como Sagüés:

a) Los jueces y tribunales internos están sujetos al imperio de la ley y obligados a aplicar las disposiciones vigentes en el ordenamiento jurídico.

b) Cuando un Estado ha ratificado un tratado internacional como la $\mathrm{CADH}$, sus jueces, como parte del aparato del Estado, también están sometidos a esta.

c) En consecuencia, el Estado está obligado a que los efectos de las disposiciones no se vean mermadas por la aplicación de leyes contrarias a su objeto y fin. ${ }^{43}$

En resumen, el Poder Judicial debe ejercer una especie de "control de convencionalidad", que, en sí, implica una comunicación con ciertos elementos compartidos del SIDH. La siguiente sección analizará los hallazgos del levantamiento de información existente, con el fin de a) encontrar esa columna vertebral común, y b) revisar si existe un uso igual de vinculante de la Corte Interamericana.

Finalmente, hay un elemento importante a nivel filosófico de todo análisis de derecho comparado: el diálogo y uso de fuentes entre las cortes y tribunales constitucionales entre sí o con tribunales supranacionales es hoy más fluido que nunca y eso obliga a ver que la metodología comparativa nos fuerza a abandonar nuestro sentido cerrado

40. Ver Elizabeth Salmon, Curso de Derecho Internacional Público (Lima: PUCP, 2015).

41. José Manuel Sobrino, "El derecho de la integración”, THËMIS-Revista de Derecho, vol. 42 (2001): 8.

42. Santiago Basabe, Jueces sin toga: Politicas judiciales y toma de decisiones en el Tribunal Constitucional del Ecuador (1999-2007) (Quito: FLACSO Ecuador, 2011).

43. Néstor Sagüés, "Obligaciones internacionales y control de convencionalidad", Estudios constitucionales, año 8 (1) (2010): 117-36. 
del ordenamiento jurídico, ${ }^{44} \mathrm{y}$ en consecuencia la posibilidad de la construcción de un sistema jurídico más afinado y basado en elementos medibles y sujetos a prueba.

\section{EL ANÁLISIS DE REDES SOCIALES COMO HERRAMIENTA DEL DERECHO: PROPUESTA METODOLÓGICA}

Uno de los intereses más importantes de las últimas tres décadas, es la investigación de las relaciones y la colaboración en las actividades humanas. En respuesta, el análisis de redes sociales (SNA) surgió ofreciendo herramientas para explorar estas relaciones en profundidad. ${ }^{45}$ Esta técnica permite capturarlas de una manera más matizada, centrándose en los patrones y cualidades de las relaciones. ${ }^{46}$ En este sentido la teoría propone lo siguiente: ${ }^{47}$

a) Cada tipo de construcción social se puede representar en términos de unidades que componen esta agregación y relaciones entre estas unidades. Este tipo de representación de una estructura social se llama "Red Social".

b) En una red social, cada unidad, generalmente llamada "actor social" (una persona, un grupo, una organización, una nación, o en el caso del presente texto, una sentencia), se representa como un nodo. Una relación se representa como un enlace o arista entre estos nodos.

c) El conjunto de relaciones posibles es potencialmente infinito; el término relación puede tener muchos significados diferentes.

Esas conexiones pueden representarse de forma matemática mediante representaciones de las conexiones; y gráficamente, mediante la tecnología de la información. ${ }^{48}$ En ese sentido, la siguiente sección hará un acercamiento que busca demostrar la existencia de conexiones (gracias a sentencias del SIDH) entre las sentencias de tres jurisdicciones, con el fin de encontrar aquellas que constituyen clusters de información (puntos en que se unen varias sentencias de las Cortes). De existir un Ius Commune,

44. Ver María Dolores Collazos, “¿Por qué comparar? Propósitos y desafíos del derecho constitucional comparado en el siglo XXI", Precedente. Revista Jurídica 18 (2021): 11-39.

45. Charu Aggarwal, "An Introduction to Social Network Data Analytics", en Social Network Data Analytics, ed. Charu Aggarwal (Boston, MA: Springer, 2011), 1-15.

46. Ver Wasserman y Faust, Social network analysis.

47. Martino Francesco y Andrea Spoto, "Social network analysis: a brief theoretical review and further perspectives in the study of information technology", PsychNology Journal, vol. 4 (1) (2006): 53-86.

48. Reza Yousefi Nooraie et al., "Social network analysis: An example of fusion between quantitative and qualitative methods", Journal of Mixed Methods Research, vol. 14 (1) (2020): 1-15, doi:/10.1177/1558689818804060. 
debería existir características estructurales de la red en estos clústeres, conexiones y elementos comunes. ${ }^{49}$

\section{ANÁLISIS: REDES JURISPRUDENCIALES DE LAS CORTES CONSTITUCIONALES ANDINAS}

En este apartado se explicarán las herramientas metodológicas usadas para explicar el comportamiento de tres jurisdicciones (Ecuador, Perú y Colombia) con relación a la respuesta de sus Cortes Constitucionales respecto al uso de la jurisprudencia, comprobar si usan de forma obligatoria los elementos existentes en la misma, y si existen elementos comunes. Se eligieron estas tres jurisdicciones por ser parte de un eje regional, han tenido crisis respecto a los temas más críticos del quehacer constitucional, y han usado la jurisprudencia del CIDH para profundos cambios en la cultura jurídica del país. ${ }^{50}$ En este orden, el análisis mixto se basará en los siguientes supuestos:

\section{UNIVERSO DE ESTUDIO}

Como universo de estudio, se usarán sentencias de las Cortes Constitucionales de las Repúblicas del Ecuador, Colombia y Perú realizadas durante el año 2020. Estas tres jurisdicciones son la unidad de análisis pues usan la jurisprudencia del SIDH obligatoria en el sistema y cuentan con cláusulas de apertura y aplicación directa de las mismas. ${ }^{51}$ Se usarán como nodos sentencias de SIDH y aquellas de los diferentes Tribunales, de forma que se puedan demostrar a) si todas usan jurisprudencia de la

49. Alexandra Marin y Barry Wellman, "Social network analysis: An introduction", en The SAGE handbook of social network analysis, eds. John Scott y Peter Carrington (Thousand Oaks: Sage, 2011), 11-25, doi:10.4135/9781446294413.n2.

50. Ver María Carmelina Londoño Lázaro, "La efectividad de los fallos de la Corte Interamericana de Derechos Humanos", Díkaion 19 (14) (2005): 203-8.

51. Se aceptan como criterio hermenéutico relevante en derechos fundamentales (S. C-327/16), y Criterio hermenéutico relevante para establecer sentido de derechos fundamentales (S. C-010/00, C-500/14). En el caso ecuatoriano es aceptada mediante los artículos 424 y 425 de nuestra Constitución, y en las sentencias Sentencia: n. ${ }^{\circ}$ 009-16-DTI-CC, del 12 de abril de 2016, MP: DR. Principales PML Pamela Martínez Loayza, Registro Oficial n. ${ }^{\circ}$ 900, Suplemento, 12 de diciembre de 2016, y Sentencia n. ${ }^{\circ} 132-16$-SEP-CC, del 20 de abril de 2016, MP: DR. Principales WMA Wendy Molina Andrade, Registro Oficial n. ${ }^{\circ}$ 799, Suplemento, 18 de julio de 2016. En Perú la cita es mucho más patente:

“... La vinculatoriedad de las sentencias de la Corte Interamericana no se agota en su parte resolutiva (la cual, ciertamente, alcanza solo al Estado que es parte en el proceso), sino que se extiende a su fundamentación o ratio decidendi, con el agregado de que, por imperio de la [Cuarta Disposición Final y Transitoria] CDFT de la Constitución y el artículo V del Título Preliminar del Código Procesal Constitucional, en 
Corte; b) cuáles son las sentencias del SIDH compartidas en esas jurisdicciones; y c) entender las razones del uso de esas normas en particular.

Para poder visualizar las conexiones se usará el software libre Gephi, trasladando esta información de manera automatizada a través de un algoritmo probado, de forma que se pueda realizar la representación gráfica de la red..$^{52}$ La ventaja de usar este software es su potencia como herramienta interactiva utilizada para la visualización y el examen de redes. ${ }^{53}$ El diseño de red se basará en el algoritmo Fruchterman-Reingold que fuerza nodos fuertemente correlacionados más cerca entre sí. Esta heurística busca generar un sistema de centralidad de los nodos más usados, de forma "simple, elegante, conceptualmente intuitiva y eficiente". ${ }^{54}$

\section{DATOS OBTENIDOS}

La siguiente sección indicará los datos obtenidos. En primer lugar, al revisar las sentencias aparecidas en los boletines de relatoría de las tres cortes, se ve una apreciación importante: el porcentaje de uso de la jurisprudencia del SIDH es marginal, de acuerdo con la data resumida en la siguiente tabla:

Figura 1. Uso de jurisprudencia del Sistema Interamericano

\begin{tabular}{cccc}
\hline País & Número de sentencias & $\begin{array}{c}\text { Sentencias con cita } \\
\text { del SIDH }\end{array}$ & $\begin{array}{c}\text { Porcentaje de uso } \\
\text { de sentencias SIDH }\end{array}$ \\
\hline Ecuador & 137 & 22 & 16,06 \\
Colombia & 171 & 30 & 17,54 \\
Perú & 35 & 10 & 28,57 \\
\hline
\end{tabular}

Fuente y elaboración propia.

dicho ámbito la sentencia resulta vinculante para todo poder público nacional, incluso en aquellos casos en los que el Estado peruano no haya sido parte en el proceso".

52. Eric Rice y Amanda Yoshioka-Maxwell, "Social Network Analysis as a Toolkit for the Science of Social Work", Journal of the Society for Social Work and Research, vol. 6 (3) (2015): 376, doi:10.1086/682723.

53. Ahsan Wajahat et al., "Interactively Visualize and Analyze Social Network Gephi" (ponencia presentada en el 3rd International Conference on Computing, Mathematics and Engineering Technologies (iCoMET), Sukkur, Pakistán, 29 a 30 de enero de 2020.

54. Thomas Fruchterman y Edward Reingold, "Graph drawing by force-directed placement", Softw. Pract. Exp., vol. 21 (11) (1991): 1159-60, doi:10.1002/spe.4380211102. 
Eso no implica que se pueda asumir que no se utilicen los estándares del SIDH, ya que no se ha hecho una búsqueda retrospectiva de las diferentes aplicaciones previas de la jurisprudencia. Debe tenerse en cuenta que no hay un uso estandarizado de boletines en las tres jurisdicciones, por tanto, son decisiones que pudieran tener elementos discordes, y cada jurisdicción tiene un sistema distinto de tratar y calificar a su set propio de acciones constitucionales. Pero lo que si se debe tener en cuenta es que, a pesar de que son obligatorias de aplicación dentro del sistema, al ser parte del corpus iure internacional, no aparecen al menos, en la mayor parte de fallos.

La situación también es interesante al analizar las sentencias aplicables. La red levantada sobre la relación jurisprudencial es la siguiente:

Figura 2. Red de relación jurisprudencial \#13A

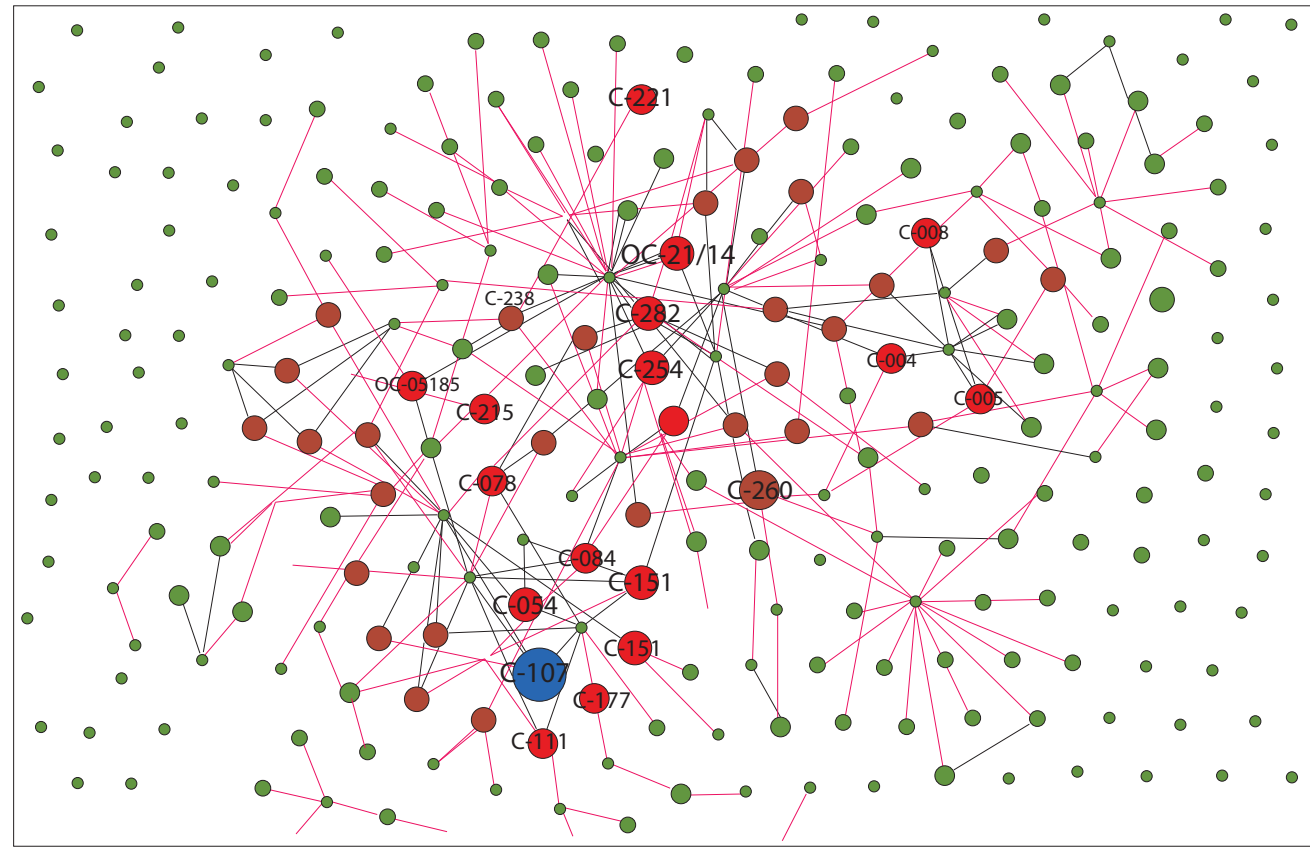

Fuente: Levantamiento de información de Sentencias Constitucionales graficada en Gephi mediante distribución Fructerman-Reinghold.

Elaboración propia.

Como se ve, existe un elemento importante: hay nodos (sentencias del SIDH) que tienen mayor preponderancia. Las sentencias con mayor grado de entrada, es decir 
aquellas que los actores consideran prominentes porque muchos actores mantienen enlaces directos con ellos son los siguientes: ${ }^{55}$

Figura 3. Jurisprudencia compartida del SIDH en Cortes Constitucionales de Ecuador Perú y Colombia

\begin{tabular}{|c|c|c|}
\hline Caso & Nombre del caso & Resumen \\
\hline C-107 & $\begin{array}{l}\text { Corte IDH. Caso Herrera Ulloa vs. } \\
\text { Costa Rica. Sentencia de } 2 \text { de julio de } \\
\text { 2004. Serie C n. }{ }^{\circ} 107 .\end{array}$ & $\begin{array}{l}\text { Responsabilidad internacional del Estado por } \\
\text { la imposición de una condena por difamación } \\
\text { en perjuicio de Mauricio Herrera Ulloa y la } \\
\text { falta de un recurso adecuado y efectivo para } \\
\text { cuestionar dicha medida. }\end{array}$ \\
\hline OC-21 & $\begin{array}{l}\text { Corte IDH. Derechos y garantías de } \\
\text { niñas y niños en el contexto de la mi- } \\
\text { gración y/o en necesidad de protección } \\
\text { internacional. Opinión Consultiva } \\
\text { OC-21/14 de } 19 \text { de agosto de } 2014 . \\
\text { Serie A n. }{ }^{\circ} 21 .\end{array}$ & $\begin{array}{l}\text { Derechos y garantías de niñas y niños en el } \\
\text { contexto de la migración y/o en necesidad de } \\
\text { protección internacional. }\end{array}$ \\
\hline C-282 & 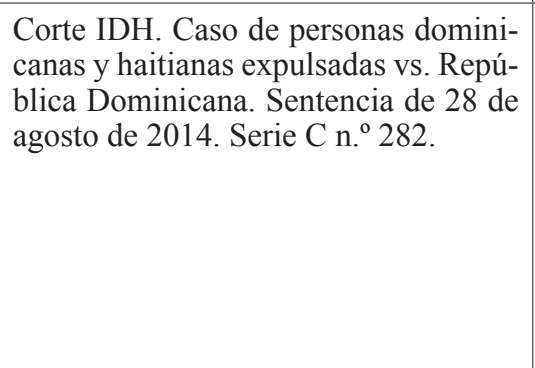 & $\begin{array}{l}\text { El Estado de República Dominicana expulsó } \\
\text { de su país a un grupo de personas de origen } \\
\text { haitiano, aun cuando algunas de estas habían } \\
\text { nacido en territorio dominicano. La Corte con- } \\
\text { cluyó que dichas expulsiones violaron los si- } \\
\text { guientes derechos: reconocimiento de la perso- } \\
\text { nalidad jurídica, integridad personal, libertad } \\
\text { personal, protección de la honra y la dignidad, } \\
\text { protección de la familia, nacionalidad, dere- } \\
\text { chos del niño y debido proceso. }\end{array}$ \\
\hline $\mathrm{C}-254$ & $\begin{array}{l}\text { Corte IDH. Caso Atala Riffo y niñas } \\
\text { vs. Chile. Sentencia de } 21 \text { de noviem- } \\
\text { bre de } 2012 \text {. Serie } \mathrm{C} \mathrm{n} .^{\mathrm{o}} 254 \text {. }\end{array}$ & $\begin{array}{l}\text { Responsabilidad internacional del Estado por } \\
\text { el trato discriminatorio y la interferencia arbi- } \\
\text { traria en la vida privada y familiar de Karen } \\
\text { Atala Riffo, debido a su orientación sexual, en } \\
\text { el proceso judicial que resultó en el retiro del } \\
\text { cuidado y custodia de sus hijas M., V. y R. }\end{array}$ \\
\hline C- 054 & $\begin{array}{l}\text { Corte IDH. Caso Ivcher Bronstein vs. } \\
\text { Perú. Fondo, Sentencia de } 6 \text { de febrero } \\
\text { de } 2001 \text {. Serie } \mathrm{C} \text { n. }{ }^{\circ} 74 \text {. }\end{array}$ & $\begin{array}{l}\text { Responsabilidad internacional del Estado pe- } \\
\text { ruano por la pérdida de nacionalidad peruana } \\
\text { de Baruch Ivcher Bronstein y la pérdida de sus } \\
\text { acciones de un canal de televisión. }\end{array}$ \\
\hline C-151 & $\begin{array}{l}\text { Corte IDH. Caso Claude Reyes y otros } \\
\text { vs. Chile. Fondo, Sentencia de } 19 \text { de } \\
\text { septiembre de } 2006 \text {. Serie C n. }{ }^{\circ} 151 .\end{array}$ & $\begin{array}{l}\text { Responsabilidad internacional por la nega- } \\
\text { tiva de brindar información relacionada a un } \\
\text { proyecto de industrialización forestal al señor } \\
\text { Marcel Claude Reyes, así como a la falta de un } \\
\text { recurso adecuado y efectivo para cuestionar tal } \\
\text { decisión. }\end{array}$ \\
\hline
\end{tabular}

55. Ver Wasserman y Faust, Social network analysis. 


\begin{tabular}{|c|c|c|}
\hline Caso & Nombre del caso & Resumen \\
\hline C-260 & $\begin{array}{l}\text { Corte IDH. Caso Mendoza y otros vs. } \\
\text { Argentina. Sentencia de } 14 \text { de mayo } \\
\text { de } 2013 \text {. Serie } \mathrm{C} \mathrm{n} .^{\circ} 260 \text {. }\end{array}$ & $\begin{array}{l}\text { Responsabilidad internacional del Estado por } \\
\text { la imposición de la prisión y reclusión perpe- } \\
\text { tuas a menores de } 18 \text { años, y a los recursos in- } \\
\text { terpuestos contra las sentencias condenatorias. }\end{array}$ \\
\hline $\mathrm{C}-221$ & $\begin{array}{l}\text { Corte IDH. Caso Gelman vs. Uruguay. } \\
\text { Sentencia de } 24 \text { de febrero de } 2011 . \\
\text { Serie C n. }{ }^{\circ} 221 .\end{array}$ & $\begin{array}{l}\text { El caso se refiere a la responsabilidad interna- } \\
\text { cional del Estado por la desaparición forzada } \\
\text { de María Claudia García Iruretagoyena de Gel- } \\
\text { man, así como de la supresión y sustitución de } \\
\text { identidad de María Macarena Gelman García. }\end{array}$ \\
\hline
\end{tabular}

Fuente y elaboración propia.

\section{DISCUSIÓN DE LA DATA}

En virtud de lo expuesto en los párrafos precedentes, surgen las siguientes dudas: ¿cómo funciona el sistema jurídico para elegir una causa vinculante? ¿Cómo discriminar entre una situación jurídica y otra? ¿Las sentencias con mayor uso, son usadas de forma equivalente en las tres jurisdicciones? En la norma y en la jurisprudencia de la Corte IDH se dice que es un caso que haya sucedido antes, pero al carecer de una regla de stare decisis, no existe nada que obligue las Cortes utilizar un precedente y no otro. En segundo lugar, no hay ninguna obligación legal o jurisprudencial que pueda mostrar de forma evidente la elección que debe hacer el juez.

Para determinar esta línea de conducta, se aplicará la técnica de la línea jurisprudencial, a través de un problema jurídico. Para proceder con determinada técnica resulta conveniente graficarla, lo cual se explica de la siguiente manera:

Una idea jurisprudencial es una idea abstracta. Para ayudar a "ver" la línea jurisprudencial resulta conveniente tratar de graficarla. Puesta sobre un gráfico, una línea de jurisprudencia es una pregunta o problema jurídico bien definido, bajo el cual se abre un espacio de posibles respuestas. En este espacio abierto, con todas las posibles respuestas a la pregunta planteada, es una estrategia conveniente para graficar las soluciones que la jurisprudencia ha dado al problema y para reconocer, si existe, un patrón de desarrollo decisional. En campo abierto que generan las dos respuestas extremas posibles hace que la línea sea, en sus extremos, bipolar. ${ }^{56}$

En este estudio de partida, se observa (en este primer acercamiento) que no existe: a) un sistema racional de elección de jurisprudencia por las Cortes Nacionales; y b)

56. Diego López Medina, El derecho de los jueces (Bogotá: Legis, 2002). 
no existe un elemento que demuestre que los casos que se comparten entre las tres jurisdicciones pueden formar una línea de elementos comunes que sean parte de un "derecho constitucional común". Esa detección es contra fáctica, y debería entenderse más que del consenso de técnica jurídica, sino de la aceptación política de que: a) no existe una regla que obliga a usar los caso del SIDH y que no se infiere qué casos se escogen para solucionar una determinada temática; y b) por qué se eligen esos casos y se rechazan otros. Esto es importante, ya que se supone que toda decisión se basa en pasos interdependientes y acumulativos de una elección racional. ${ }^{57}$ Sin una regla racional, algunos autores proponen que la decisión de "qué jurisprudencia usar" controla a las partes en el caso, y resolver casos futuros, así que se evita usar fuentes que puedan usarse en otras causas, o usarlas para generar legitimidad. ${ }^{58}$

Por tanto, en un escenario como nuestras cortes, con un stare decisis inexistente o limitado a su propia jurisprudencia, sin una regla de aplicación se generan situaciones en que la acción del juez tiene limitaciones: al no estar obligado a revisar las herramientas jurídicas a su cargo - que se supone son obligatorias - aplica en función de su conocimiento previo personal. Se espera que los tribunales, especialmente los de más alta instancia, tomen en cuenta las decisiones anteriores y superiores cuando existe un nivel suficiente de coherencia en la jurisprudencia.

\section{CONCLUSIONES}

El presente texto trata de demostrar con ciertos datos un fenómeno excepcional: la ampliación y modernización de todo el sistema de jurisprudencia de las cortes constitucionales, a través de un diálogo y aplicación de normas del Sistema Interamericano de Derechos Humanos, el ius Commune latinoamericano. El estudio demuestra inicialmente que, aunque las jurisdicciones de los países andinos no tienen una regla racional de uso de la jurisprudencia: a) existe un esfuerzo de usar la jurisprudencia del SIDH en los tribunales constitucionales; y b) a pesar de la existencia de elementos de bloque de constitucionalidad en las tres jurisdicciones, no existe una lógica de aplicación de esa herramienta en los países del área andina.

En este sentido, es interesante (para efectos de estudios futuros), la posibilidad de utilizar la metodología de redes sociales como un mecanismo en el que se construyen formas que permitan hacer una medición cuali-cuantitativa, con la suficiente capa-

57. Rafael Bañon, La nueva administración pública (Madrid: Alianza Editorial, 1997), 292.

58. Howard Wasserman, "Precedent, Particularized Injunctions, and Judicial Departmentalism: A Model of Constitutional Adjudication", Lewis \& Clark Law Review, n. . 19-02 (2020). 
cidad para explicar las zonas grises supuestas en el texto. Esta situación demuestra que el SNA puede servir como una herramienta no solo para medir relaciones, sino influencias, y prospectivas mediante estudios agregados. ${ }^{59}$ Un siguiente paso, sería analizar el cómo y el por qué: si estas sentencias se construyen como un ejercicio estático para casos concretos, o si realmente la interpretación sostenida por los las Altas Cortes a) corresponde a un tema determinado; y b) se sostienen a lo largo del tiempo, con lo que se puede identificar el "patrón fáctico fundamental y relacionarlo con el texto o norma constitucional controlante". ${ }^{60}$

Finalmente, debe pensarse en generar estudios transversales a nivel cuantitativo de los estudios de las fuentes del Derecho, ya que, una de las complicaciones de la materia es un ejercicio de continua interpretación de la norma, lo que puede llevar a que se generen opiniones jurídicas subyacentes que no son entendidas ni construidas por los actores del sistema jurídico, ${ }^{61}$ por tanto, debe empezarse a generar aumento de objetividad en el análisis de los fenómenos jurídicos y, en consecuencia, la posibilidad de la construcción de un sistema jurídico más afinado y basado en elementos medibles y sujetos a prueba.

\section{BIBLIOGRAFÍA}

Aggarwal, Charu. "An Introduction to Social Network Data Analytics". En Social Network Data Analytics, editado por Charu Aggarwal, 1-15. Boston, MA: Springer, 2011.

Aragón Reyes, Manuel. “Dos problemas falsos y uno verdadero: 'neoconstitucionalismo', 'garantismo’ y aplicación judicial de la Constitución”. Cuestiones Constitucionales, n. ${ }^{\circ} 29$ (2015): 3-25. doi:10.1016/s1405-9193(13)71288-5.

Ávila, Ramiro. El neoconstitucionalismo transformador. El Estado y el derecho en la Constitución de 2008. Quito: Universidad Andina Simón Bolívar, Sede Ecuador, 2011.

Bañon, Rafael. La nueva administración pública. Madrid: Alianza Editorial, 1997.

Barbosa Delgado, Francisco. "Los límites a la doctrina del margen nacional de apreciación en el Tribunal Europeo y la Corte Interamericana de Derechos Humanos: intervención judicial en torno a ciertos derechos de las minorías étnicas y culturales". Revista Derecho del Estado, n. ${ }^{\circ} 26$ (2011): 107-35. https://revistas.uexternado.edu.co/index.php/derest/article/ view/2881.

59. Julio César Rodríguez Treviño, "Cómo utilizar el Análisis de Redes Sociales para temas de historia". Signos históricos, vol. 15 (29) (2013): 102-41.

60. López Medina, El derecho de los jueces, 62.

61. Duncan Kennedy, La enseñanza del Derecho como forma de acción política (Buenos Aires: Siglo XXI, 2012). 
Basabe, Santiago. Jueces sin toga: Politicas judiciales y toma de decisiones en el Tribunal Constitucional del Ecuador (1999-2007). Quito: FLACSO Ecuador, 2011.

Carozza, Paolo. “' 'My Friend is a Stranger': The Death Penalty and the Global Ius Commune of Human Rights". Texas Law Review, vol. 81 (2003): 1031-86. https://scholarship.law. nd.edu/law_faculty_scholarship/536.

Castilla Juárez, Karlos. “¿Qué tan común es lo común del Ius Constitutionale Commune Latinoamericano?". Ventana Jurídica, 21 de junio de 2017. http://facultad.pucp.edu.pe/derecho/blog/que-tan-comun-es-lo-comun-del-ius-constitutionale-commune-latinoamericano/.

Collazos, María Dolores. “¿Por qué comparar? Propósitos y desafíos del derecho constitucional comparado en el siglo XXI”. Precedente. Revista Jurídica 18 (2021): 11-39.

Conde y Luque, Rafael. Derecho internacional público. Sevilla: Athenaica Ediciones Universitarias, 2015.

Corte IDH. "Sentencia de 21 de noviembre de 2012 (Solicitud de Interpretación de la Sentencia de Fondo, Reparaciones y Costas)". Caso Atala Riffo y niñas vs. Chile. 21 de noviembre de 2012. http://www.corteidh.or.cr/docs/casos/articulos/seriec_254_esp.pdf.

—. "Sentencia de 19 de septiembre de 2006 (Fondo, Reparaciones y Costas)". Caso Claude Reyes y otros vs. Chile. 19 de septiembre de 2006. https:/www.corteidh.or.cr/docs/casos/ articulos/seriec_151_esp.pdf.

—. "Sentencia de 6 de febrero de 2020 (Fondo, Reparaciones y Costas)". Caso Comunidades Indígenas Miembros de la Asociación Lhaka Honhat (Nuestra Tierra) vs. Argentina. 6 de febrero de 2020. http://www.corteidh.or.cr/docs/casos/articulos/seriec_400_esp.pdf.

—. "Sentencia de 15 de septiembre de 2005 (Fondo, Reparaciones y Costas)". Caso de la "Masacre de Mapiripán” vs. Colombia. 15 de septiembre de 2005. http://www.corteidh. or.cr/docs/casos/articulos/seriec_134_esp.pdf.

—. "Sentencia de 28 de agosto de 2014 (Excepciones Preliminares, Fondo, Reparaciones y Costas.)". Caso de Personas dominicanas y haitianas expulsadas vs. República Dominicana. 28 de agosto de 2014. http://corteidh.or.cr/docs/casos/articulos/seriec_282_esp.pdf.

—. "Sentencia de 24 de febrero de 2011(Fondo y Reparaciones)". Caso Gelman vs. Uruguay. 24 de febrero de 2011. https://www.corteidh.or.cr/docs/casos/articulos/seriec_221_esp1. pdf.

—. "Sentencia de 2 de julio de 2004 (Excepciones Preliminares, Fondo, Reparaciones y Costas)". Caso Herrera Ulloa vs. Costa Rica. 2 de julio de 2004. https://www.corteidh.or.cr/ docs/casos/articulos/seriec_107_esp.pdf.

—. "Sentencia de 6 de febrero de 2001 (Fondo, Reparaciones y Costas)". Caso Ivcher Bronstein vs. Perú. 6 de febrero de 2001. http://www.corteidh.or.cr/docs/casos/articulos/Seriec_74_esp.pdf.

—. "Sentencia de 14 de mayo de 2013 (Excepciones Preliminares, Fondo y Reparaciones)". Caso Mendoza y otros vs. Argentina. 14 de mayo de 2013. https://www.corteidh.or.cr/docs/ casos/articulos/seriec_260_esp.pdf. 
—. "Sentencia de 5 de febrero de 2018 (Excepciones Preliminares, Fondo, Reparaciones y Costas)". Caso Pueblo Indígena Xucuru y sus miembros vs. Brasil. 5 de febrero de 2018. https://www.corteidh.or.cr/docs/casos/articulos/seriec_346_esp.pdf.

—. "Sentencia de 20 de octubre de 2016 (Excepciones Preliminares, Fondo, Reparaciones y Costas)". Caso Trabajadores de la Hacienda Brasil Verde vs. Brasil. 20 de octubre de 2016. http://www.corteidh.or.cr/docs/casos/articulos/seriec_318_esp.pdf.

—. "Opinión Consultiva OC-21/14 de 19 de agosto de 2014”. Derechos y garantías de niñas y niños en el contexto de la migración y/o en necesidad de protección internacional. 19 de agosto de 2014.

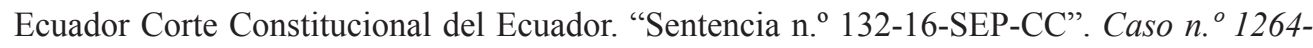
15-EP, 20 de abril de 2020.

Ferrajoli, Luigi. Poderes salvajes: la crisis de la democracia constitucional. Madrid: Trotta, 2013.

Francesco, Martino, y Andrea Spoto. "Social network analysis: a brief theoretical review and further perspectives in the study of information technology". PsychNology Journal, vol. 4 (1) (2006): 53-86.

Fruchterman, Thomas, y Edward Reingold. "Graph drawing by force-directed placement". Softw. Pract. Exp., vol. 21 (11) (1991): 1129-64. doi:10.1002/spe.4380211102.

García Jaramillo, Leonardo. “De la ‘constitucionalización' a la 'convencionalización' del ordenamiento jurídico. La contribución del ius constitutionale commune". Revista Derecho del Estado, n. 36 (2016): 131-66. doi:10.18601/01229893.n36.05.

García Ramírez, Sergio. “La 'navegación americana' de los derechos humanos: hacia un Ius Commune". En Ius Constitutionale Commune en América Latina. Texto básico para su comprensión, coordinado por Armin von Bogdandy, Mariela Morales Antoniazzi y Eduardo Ferrer Mac-Gregor, 55-190. Querétaro: Max Planck Institute for Comparative Public Law and International Law, 2017.

Jellinek, Georg. Teoría General del Estado. Buenos Aires: Albatros, 1978.

Johnson, Burke, Anthony Onwuegbuzie y Lisa Turner. "Toward a Definition of Mixed Methods Research". Journal of Mixed Methods Research, vol. 1 (2) (2007): 112-33. doi:10.1177/1558689806298224.

Kennedy, Duncan. La enseñanza del Derecho como forma de acción política. Buenos Aires: Siglo XXI, 2012.

Keohane, Robert, y Joseph Nye. Power and Interdependence. World Politics in Transition. Boston: Little Brown and Company, 1977.

Kissinger, Henry. Orden Mundial. Barcelona: Debate, 2016.

Korobkin, Russell, y Thomas Ulen. "Law and behavioral science: Removing the rationality assumption from law and economics". California Law Review, vol. 88 (2000): 1053-66. doi: $10.2139 /$ ssrn.229937.

Lee, Jootaek. "Paradox of Hierarchy and Conflicts of Values: International Law, Human Rights, and Global Governance". Northwestern University Journal of International Human Rights, vol. 18 (1) (2019): 75-94. 
Londoño Lázaro, María Carmelina. "La efectividad de los fallos de la Corte Interamericana de Derechos Humanos". Díkaion 19 (14) (2005): 203-8.

López Medina, Diego. El derecho de los jueces. Bogotá: Legis, 2002.

Lopez-Claros, Augusto, Arthur Dahl y Maja Groff. Global Governance and the Emergence of Global Institutions for the 21st Century. Nueva York: Cambridge University Press, 2020.

Marin, Alexandra, y Barry Wellman. "Social network analysis: An introduction". En The SAGE handbook of social network analysis, editado por John Scott y Peter Carrington, 1125. Thousand Oaks: Sage, 2011. doi:10.4135/9781446294413.n2.

Molero Martínez, Ney. "Justicia constitucional y democracia: casos Colombia, Ecuador y Venezuela”. Revista Digital de Investigación y Postgrado, vol. 2 (3) (2021): 89-100.

Morales Antoniazzi, Mariela. “¿La democracia como principio del ius constitutionale commune en América Latina? Construcción, reconstrucción y desafíos actuales para la justicia constitucional”. En Ius Constitutionale Commune en América Latina. Texto básico para su comprensión, coordinado por Armin von Bogdandy, Mariela Morales Antoniazzi y Eduardo Ferrer Mac-Gregor, 417-57. Querétaro: Max Planck Institute for Comparative Public Law and International Law, 2017.

Oyarte, Rafael. Derecho Constitucional. Quito: Corporación de Estudios y Publicaciones, 2015.

Pampillo Baliño, Juan Pablo. "Derecho comunitario y ius commune americano: dos asignaturas pendientes para las ciencias jurídicas de nuestro continente". Foro: Revista de Derecho, n. ${ }^{\circ} 18$ (2017): 5-29.

Pittier, Lautaro, y Ricardo Rincón. "La evolución de los DDHH en la Constitución. Diálogos y tensiones entre la Constitución Formal y la Constitución Material". Revista Argentina de Derecho Público, n. 5 (2020).

Remiro Brotóns, Antonio. Derecho Internacional. Valencia: Tirant lo Blanch, 2007.

Rice, Eric, y Amanda Yoshioka-Maxwell. "Social Network Analysis as a Toolkit for the Science of Social Work". Journal of the Society for Social Work and Research, vol. 6 (3) (2015): 369-83. doi:10.1086/682723.

Rodríguez Treviño, Julio César. "Cómo utilizar el Análisis de Redes Sociales para temas de historia”. Signos históricos, vol. 15 (29) (2013): 102-41.

Sagüés, Néstor. “Obligaciones internacionales y control de convencionalidad”. Estudios constitucionales, año 8 (1) (2010): 117-36.

Salmon, Elizabeth. Curso de Derecho Internacional Público. Lima: PUCP, 2015.

Sánchez-Molina, Pablo. "Margen de apreciación nacional (en los sistemas de protección internacional de los derechos humanos)". EUNOMÍA. Revista en Cultura de la Legalidad, n. ${ }^{\circ}$ 9 (2016): 224-31.

Sandholtz, Wayne, y Mariana Rangel Padilla. "Law and Politics in the Inter-American System: The Amnesty Cases". Journal of Law and Courts 8 (1) (2020): 10.

Santano, Ana. "Derechos humanos para el desarrollo de una sociedad realmente globalizada". Opinión Jurídica 19 (38) (2020): 39-57. 
Sobrino, José Manuel. "El derecho de la integración”. THĒMIS-Revista de Derecho, vol. 42 (2001): 7-27.

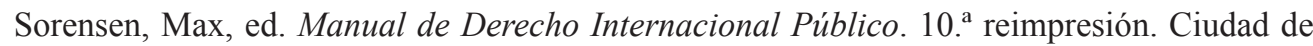
México: Fondo de Cultura Económica, 2008.

Spura, Fabián, y Efrén Guerrero. "Polarización política: análisis de la relación interpartidista en Twitter. Acercamiento al caso comparado España. Ecuador". Ponencia presentada en el XII congreso de AECPA Euskal Herriko Unibertsitatea-Universidad del País Vasco, San Sebastián, 13 a 15 de junio de 2015.

Von Bogdandy, Armin. "Ius Constitutionale Commune in Latin America: A Look at a Transformative Constitutionalism". Revista Derecho del Estado, n. ${ }^{\circ} 34$ (2015): 3-50.

Von Bogdandy, Armin, y Pedro Villarreal. International Law on Pandemic Response: A First Stocktaking in Light of the Coronavirus Crisis. Heidelberg: Max Planck Institute for Comparative Public Law \& International Law (MPIL), 2020.

Wajahat, Ahsan, Nazir Ahsan, Akhtar Faheem, Qureshi Sirajuddin, Ulah Faheem, Razaque Fahad y Shakeel Anum. "Interactively Visualize and Analyze Social Network Gephi”. Ponencia presentada en el 3rd International Conference on Computing, Mathematics and Engineering Technologies (iCoMET), Sukkur, Pakistán, 29 a 30 de enero de 2020.

Wasserman, Howard. "Precedent, Particularized Injunctions, and Judicial Departmentalism: A Model of Constitutional Adjudication”. Lewis \& Clark Law Review, n. ${ }^{\circ} 19-02$ (2020).

Wasserman, Stanley, y Katherine Faust. Social network analysis: Methods and applications. Nueva York: Cambridge University Press, 1994.

Yousefi Nooraie, Reza, Joanna Sale, Alexandra Marin y Lori Ross. "Social network analysis: An example of fusion between quantitative and qualitative methods". Journal of Mixed Methods Research, vol. 14 (1) (2020): 1-15. doi:/10.1177/1558689818804060. 